\title{
Consideraciones del Comité Asesor en Vacunas y Estrategias de Vacunación (CAVEI) en relación con los servicios de vacunación durante la emergencia sanitaria COVID-19
}

\author{
Considerations of the Advisory Committee on Vaccines and Vaccination Strategies \\ (CAVEI) in relation to vaccination services during the health emergency COVID-19
}

\author{
Comité Asesor en Vacunas y Estrategias de Vacunación
}

Recibido: 6 de mayo de 2020; Aceptado: 5 de junio de 2020

La entrega de servicios de inmunización programáticos por parte del Programa Nacional de Inmunizaciones busca brindar protección a la población residente en Chile frente a enfermedades prevenibles por vacunas (EPV), lo que constituye a su vez la misión del Departamento de Inmunizaciones de Chile ${ }^{1}$. La actual pandemia de SARS-CoV-2 impone desafíos a la entrega de servicios de inmunización programáticos que se alojan en las propias medidas de prevención de transmisión, como el distanciamiento social y las cuarentenas voluntaria o total, y en la potencial futura sobrecarga del sistema de salud por causa de COVID-19. La interrupción de los servicios de inmunización, por breve que sea, produce un aumento de personas susceptibles a enfermar por EPV y, consecuentemente, incrementa la probabilidad de brotes epidémicos de EPV proclives a ello, como el sarampión, cuya morbilidad y mortalidad asociadas afectan principalmente a lactantes y otros grupos vulnerables ${ }^{2}$.

El 26 de marzo de 2020, la Organización Mundial de la Salud publicó Guiding principles for immunizaton activites during the COVID-19 pandemic ${ }^{2}$, guía interina que entrega los principios rectores para la implementación de actividades de inmunización bajo la emergencia sanitaria por COVID-19. En la misma fecha, la Organización Panamericana de la Salud (OPS) publicó El programa de inmunización en el contexto de la pandemia de COVID-193. El CAVEI respalda y sugiere implementar recomendaciones de dichas guías.

En el complejo escenario de emergencia sanitaria frente a COVID-19 en que el país se encuentra y por la necesidad de procurar protección frente a EPV y así prevenir brotes a causa de ellas, el CAVEI le plantea a la autoridad sanitaria las siguientes consideraciones:

- En conocimiento del modo de transmisión de SARS-CoV-2 y de las medidas de prevención implementadas en Chile que incluyen el distanciamiento social y las cuarentenas voluntaria y total, las campañas de vacunación masiva deben ser suspendidas temporalmente. Esta medida debe ser reevaluada al ritmo del dinamismo de la situación por COVID-19. 
- Mantener la vacunación programática mientras las medidas frente a la transmisión de SARS-CoV-2 lo permitan. Difundir entre los niveles medio y ejecutor y en la población general el mensaje sobre la importancia de los servicios de vacunación como uno de los pilares de la salud pública incluso durante la pandemia de COVID-19, y reforzar que la misión del Departamento de Inmunizaciones es proteger a la población de las EPV.

- Decisiones en relación con la vacunación programática deberán tomarse en base:

$\Rightarrow$ A un análisis de la epidemiología de las EPV.

$\Rightarrow \mathrm{Al}$ escenario de transmisión y medidas de control de SARS-CoV-2.

$\Rightarrow$ A los recursos con que cuente el Departamento de Inmunizaciones para asegurar la calidad y seguridad de las vacunas e inmunizaciones, realizar monitoreo de los casos de errores programáticos y ESAVI, gestionar el cumplimiento de la cadena de frío y la distribución y compras de vacunas.

$\Rightarrow \quad \mathrm{Al}$ escenario de demanda del sistema de salud por causa de COVID-19.

- Mantener la vacunación de recién nacidos en centros hospitalarios de nacimiento según el calendario de vacunación.

- Priorizar series primarias de vacunación, especialmente vacunas que provean protección contra sarampión, rubéola y poliomielitis y enfermedad neumocóccica.

- En la campaña de vacunación contra influenza, priorizar a los grupos más vulnerables para recibir vacuna.

- Aunque actualmente no existen contraindicaciones médicas conocidas sobre vacunar a una persona con COVID-19, la OPS recomienda deferir toda vacunación hasta su completa recuperación según los criterios establecidos ${ }^{3}$.

- Aunque actualmente no existen contraindicaciones médicas conocidas sobre la vacunación de una persona que haya tenido contacto con un caso de COVID-19, la OPS recomienda diferir la vacunación hasta que se haya cumplido la cuarentena (14 días después de la última exposición) $)^{3}$.

- La implementación de vacunación programática en centros de atención primaria de salud debe realizarse:

$\Rightarrow \quad$ Sin aglomeraciones en las salas de espera, lo que podría lograrse con citaciones programadas para la vacunación de niños y adultos sanos.
$\Rightarrow$ Informar a los asistentes sobre las medidas de prevención de COVID-19, como el mantener al menos 1 metro de distancia entre personas, cubrir la nariz y boca con el pliegue del brazo al estornudar o toser, entre otras.

$\Rightarrow \quad$ Procurar la ventilación permanente de las salas de espera, aun primando el distanciamiento social dentro de ellas.

- En caso de que la emergencia sanitaria hiciera al Ministerio de Salud disponer de los recursos humanos e infraestructura de los servicios de inmunización para dirigirlos a la respuesta frente a $\mathrm{CO}$ VID-19, organizaciones civiles y organizaciones no gubernamentales con experiencia en el ámbito humanitario y de la salud podrían ser convocadas para apoyar estrategias de inmunización esenciales para mantener la protección de los grupos vulnerables frente a $\mathrm{EPV}^{4}$. Estas deberán recibir una inducción a la vacunación segura para maximizar los beneficios de la vacunación.

- En caso de brote epidémico por EPV, la implementación de una campaña de vacunación como control de brote deberá responder a un análisis de riesgo-beneficio según la EPV y la epidemiología del brote versus mayor propagación de COVID-19.

- La incorporación de nuevas vacunas al Programa Nacional de Inmunizaciones debiera aplazarse hasta después del cese de la emergencia sanitaria.

- En el escenario de que parte de la vacunación programática se vea interrumpida, los planes de puesta al día que el Departamento de Inmunizaciones desarrolle de acuerdo a las necesidades de cada cohorte requerirán de la flexibilidad presupuestaria que permita su gestión, coordinación y difusión comunicacional en la población. Lo último, para promover la demanda de vacunación y volver gradualmente a los niveles habituales para la población chilena.

Estas consideraciones del CAVEI serán revisadas y sujetas a reformulación según la situación de COVID-19 y de las enfermedades prevenibles por vacunas contempladas por el Programa Nacional de Inmunizaciones.

\section{Conflicto de intereses}

Los autores declaran no tener conflicto de intereses. 


\section{Referencias}

1. Ministerio de Salud- Gobierno de Chile. Misión del Departamento de Inmunizaciones [Internet]. Available from: https://vacunas.minsal.cl/ conozcanos/mision/

2. World Health Organization. Guiding principles for immunizaton activites during the COVID-19 pandemic. Interim guidance 26 March 2020
[Internet]. 2020. Available from: https://apps.who.int/iris/ bitstream/handle/10665/331590/WHO2019-nCoV-immunization_services2020.1-eng.pdf

3. Organización Panamericana de la Salud/ Organización Mundial de la Salud.

El programa de inmunización en el contexto de la pandemia de COVID-19 [Internet]. 2020. Available from: https://www.paho.org/es/documentos/ programa-inmunizacion-contextopandemia-covid-19-marzo-2020

4. World Health Organization Regional Office for Europe. Guidance on routine immunization services during COVID-19 pandemic in the WHO European Region [Internet]. 2020. Available from: http:// www.euro.who.int/_data/assets/ pdf_file/0004/433813/Guidance-routineimmunization-services-COVID-19pandemic.pdf 